\title{
Optimizing probes to image cleared tissue
}

\author{
Vivien Marx
}

Probes and imaging finesse for a clearer view of tissue.

Cleared tissue entices researchers to gaze deeply into their samples and tackle ambitious projects such as reconstructing neuronal circuits or characterizing a tumor from the inside out ${ }^{1-6}$. But molecular labels are not per se optimized for these tasks ${ }^{7}$. Fluorescence might be quickly quenched or too faint; immunolabels and stains can be invisible. The remedies for these problems include careful probe selection and imaging finesse (see Methagora for a compilation of experiences with various approaches; see Box 1 for a list of acronyms).

\section{Expressed fluorescence}

Fluorescence microscopy of genetically encoded fluorescent proteins (FPs) is helpful for tracking proteins in live cells and can be used with fixed, cleared tissue, too. In wellcleared tissue, if the signal is strong, FPs can be imaged, says Douglas Richardson, who directs Harvard University's Center for Biological Imaging. Among the challenges are the big data from these experiments and adapting a microscope to handle tissue that can, for example, be one centimeter thick.

But even in cleared tissue, FPs expressed at endogenous levels may not be bright enough to be seen over background, says Richardson. "The truth is, there is often still autofluorescence and scattered light," he says. In addition, preserving $100 \%$ of endogenous fluorescence of proteins in large, cleared tissue samples "is a difficult task that is not fully achieved to date by any published method in our hands," says Nicolas Renier, a postdoctoral fellow in Marc Tessier-Lavigne's lab at Rockefeller University. The signal from neurons in whole brains of Brainbow mice with genetically encoded fluorescent neurons can be too faint. The Rockefeller team has imaged small Brainbow samples 0.5 to 1 millimeter thick that were cleared with passive CLARITY, or PACT.
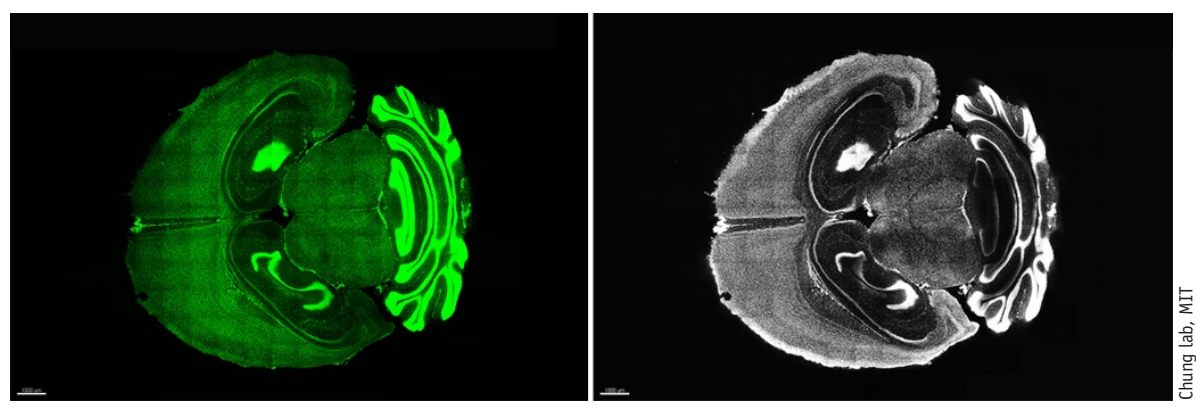

Whole mouse brain staining can take as little as one day with stochastic electrotransport. These micrographs show a brain stained with SYTO 16 (left) and an antibody to histone (right).

Fluorescence can be quenched if imaging is not done carefully or if a solvent-based clearing procedure is used, says Richardson. Most FPs require an aqueous environment to fluoresce. Solvent-based clearing dissolves lipid and dehydrates tissue, with subsequent stages of lipid removal and refractive index matching during which water would interfere. One historic solvent-based clearing agent, Spalteholz's preparation, quenches fluorescence immediately, as does iDISCO. With BABB, fluorescence lasts a few hours. Tissues cleared with 3DISCO can maintain fluorescence for a few days, which is challenging for long-term storage and for achieving consistent results in the lab, says TessierLavigne.

3DISCO was developed in the lab of HansUlrich Dodt, a researcher at the Vienna University of Technology and at the Center for Brain Research at the Medical University of Vienna. Dodt and his team are working on stabilized DISCO, or sDISCO. Preliminary imaging assays show that it handles endogenous fluorescence better, maintaining the signal from GFP and YFP, says Dodt.

Atsushi Miyawaki, now at RIKEN Brain Science Institute, was a postdoctoral fellow in the lab of Roger Tsien at the University of California at San Diego (UCSD). Miyawaki noticed that FPs still fluoresced after being exposed to high concentrations of urea, an observation that led to Scale, his urea-based clearing agent. It removes lipids with detergents rather than with organic solvents and then hydrates tissue.

A new sorbitol and urea-based version called ScaleS is gentler, says Miyawaki, and it preserves membrane structures well. Thus, for example, fluorescent proteins tethered to the plasma membrane will get along well with ScaleS, he says, which will help labs that place FPs beneath the plasma membrane to enable neuronal tracing. ScaleS will work with any fixation-resistant probes, he says. His team is using this clearing agent to compare proliferation and differentiation in fluorescent ubiquitinationbased cell cycle indicator transgenic mice.

To avoid FP quenching and to lessen scatter by making the tissue more homogeneous, labs have developed clearing agents that do not apply detergents or solvents, such as SeeDB and ClearT. But clearing large samples this way can take many weeks.

\section{Immunofluorescent labels}

Immunolabeling has been demonstrated in small samples cleared with a number of different techniques including 3DISCO, CLARITY, ClearT2, CUBIC, iDISCO, PACT/PARS, SeeDB and TDE. Wholebrain immunolabeling has been accomplished with iDISCO and CLARITY. 


\section{BOX 1 GLOSSARY OF SOME TISSUE-CLEARING AGENT ACRONYMS}

3DISC0: Three-dimensional imaging of solvent-cleared organs

BABB: Benzyl alcohol and benzylbenzoate

CLARITY: Clear lipid-exchanged acrylamide-hybridized rigid imaging/immunostaining/ in situ hybridization-compatible tissue hydrogel

CUBIC: Clear unobstructed brain imaging cocktails and computational analysis

iDISC0: Immunolabeling-enabled 3D imaging of solvent-cleared organs

iDISCO+: Immunolabeling-enabled 3D imaging of solvent-cleared organs plus

PACT: Passive clear lipid-exchanged acrylamide-hybridized rigid imaging/ immunostaining/in situ hybridization-compatible tissue hydrogel

PARS: Perfusion-assisted agent release in situ

SeeDB: See Deep Brain

Spalteholz's preparation: Benzylbenzoate-methyl salicylate

TDE: 2,2'-thiodiethanol

Pretreatment of samples with 3DISCO for immunolabeling can make them more fragile and susceptible to deformation and shrinkage, says Tessier-Lavigne. He and his team have tweaked the 3DISCO protocol to develop iDISCO+.

Getting antibody-based labels into tissue remains challenging, says Miyawaki. When labs work with large cleared tissue samples, labels must travel far. Improving antibody penetration tends to involve harsh treatments that destroy fine structures, says Takeshi Imai, a researcher at RIKEN's Center for Developmental Biology who developed SeeDB and who is working on SeeDB2. Smaller labels can help, such as single-chain antibodies or nanobodies, which are based on antibodies from camels and llamas. But, says Imai, unlike the situation for conventional antibodies, the nanobody selection in neuroscience is limited.

Other possibilities include antigenbinding fragments and aptamers, says Richardson. Labs can also amplify the signal with antibodies, choosing either the primary or the secondary antibody - or both-to be polyclonal. The signal boost comes from binding multiple antibodies to the target of interest, given that they are not all binding to the same epitope. But there is also the risk of more nonspecific labeling.

The difference in tissue density, says Richardson, is probably the key reason that results vary between labeled samples cleared with different techniques. He recommends that labs permeabilize their samples with detergent, even when working with thin tissue. "The more lipid a clearing technique removes, the better the antibody will diffuse," he says. That is why techniques that remove the most lipid, such as iDISCO and CLARITY, have proven most compatible with immunolabeling.

Tissue density can also be increased by the hydrogel used in hydrogel-embeddingbased clearing techniques, says Richardson. Immunolabeling has been shown to improve when labs increase the hydrogel's pore size, such as by reducing acrylamide cross-linking by excluding bisacrylamide from the hydrogel mix.

For experiments with cleared tissue, the beautiful and the beastly about antibody use hold true. "Each one has its own personality," says Mark Ellisman, who directs UCSD's Center for Research in Biological Systems. A given spot on a protein surface where an antibody binds might be changed upon clearing, and antigenicity can then be impaired. As Richardson says, labs must use validated antibodies and will need more antibody for abundant epitopes, and they must mind epitope availability and antibody specificity. To minimize nonspecific binding, scientists can optimize antibody concentration on smaller tissue pieces. And, says Ellisman, regardless of antibody size, labs need to flush out unbound antibody percolating through cleared tissue.

\section{Labels on the move}

When the Rockefeller team developed iDISCO, they labeled and imaged many sample types using light-sheet microscopy: the mouse brain, whole organs such as kidneys, muscle and vasculature, and entire forelimbs. They labeled both sparse and more widely expressed antigens. Overall, they found that a few days of incubation with primary antibody sufficed. They sent labels deep into the brain-for example, into the sixth cortical layer and the thalamus.

To see whether epitopes were affected by methanol, which is part of the 3 DISCO protocol, they tested 28 antibodies on tissue samples pretreated with methanol. Three antibodies proved methanol-sensitive, so they cleared without methanol to use them. Although clearing without methanol works, it extends the incubation time, because probes diffuse more slowly and there is generally higher autofluorescence. The team dissected tissue slightly to accelerate diffusion, but Renier says that the team has found that tissue trimming is not strictly necessary.

The CLARITY team, including Stanford University researchers Karl Deisseroth and Kwanghun Chung, found that they were able to maintain fluorescence with green, yellow and red FPs. With CLARITY, tissue is embedded in a hydrogel mesh and then incubated, sometimes for weeks to months, in a detergent that removes lipids.

Chung, now at MIT, explains that the team used electrophoresis to speed up the transport of detergent molecules deep into tissue for faster lipid extraction, whereas the immunolabeling involved passive incubation. It took two weeks each for a primary and a secondary antibody to penetrate 2.5 millimeters into the tissue. The team did three rounds of antibody-based labeling of mouse tissue. They noted that under stringent electrophoretic conditions, the polyacrylamide-based framework can lose some of its structural integrity. They were concerned that these conditions could affect

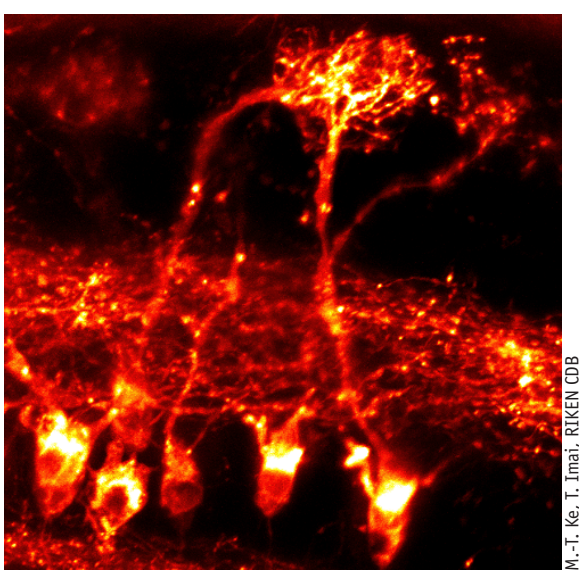

Mitral cells in the mouse olfactory bulb stained with DiI and cleared with SeeDB. 


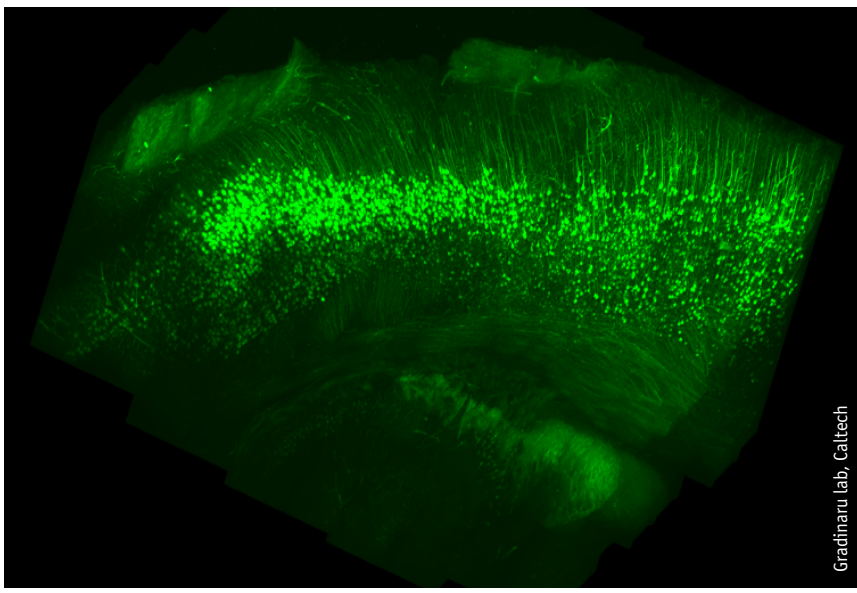

Rodent brain tissue cleared with PACT and PARS and then stored retains fluorescence of genetically encoded proteins for at least half a year. produce a gradient of strong staining on one side of the tissue that gradually decreases across the sample. The approach intrigues Tessier-Lavigne, but he believes it might be tricky to implement. He wants to see how widely it is used to fully assess the technique's impact.

Chung and his team have also developed system-wide antigenicity and hinder immunolabeling. They subsequently developed PACT to address this issue and simplify the method, says Deisseroth.

As a remedy for labels' slow penetration into cleared tissue, uneven distribution and difficulty in reaching the innermost areas of tissue samples, Chung has developed stochastic electrotransport ${ }^{8}$. Unlike the unidirectional electrical field in CLARITY's classic electrophoresis step, this approach applies a less forceful rotational electric field to accelerate probe movement and disperse probes more evenly. The team's test device positions the sample between two electrodes and then rotates it. With this device, the team found that probes with a wide range of molecular weights-antibody to histone $\mathrm{H} 3$, the nucleic acid dye SYTO 16 and a fluorophore-conjugated tomato lectin dye, which marks blood vessels-penetrated deep into a whole adult mouse brain cleared with CLARITY.

For example, without stochastic electrotransport, anti-H3 traveled around 300 micrometers into tissue, and with electrotransport it penetrated the entire depth of the cleared whole adult mouse brain in the course of one day, says Chung. The researchers had selected probes that target structures and proteins throughout the brain. When the MIT team experimented with stochastic electrotransport and samples cleared with iDISCO, probes labeled only the surface, whereas in the CUBIC-cleared mouse brain the nuclear and blood vessel stains worked throughout the tissue.

Stochastic electrotransport indeed accelerates diffusion and produces a more even distribution of the antibody within the tissue, says Richardson. It addresses issues with the electrophoretic step in CLARITY, which can control of interaction time and kinetics of chemicals, or SWITCH. With this technique, chemical reactions between endogenous tissue biomolecules and an exogenous agent, such as a fixative or a fluorescent label, are controlled by $\mathrm{pH}$ or chemical additives. A set of buffers either facilitate or block these reactions. The researchers found that SWITCH increases the penetration depth of antibodies and yields a more uniform distribution of the fluorescent signal than in a control sample. The team assumes that this is due to low levels of antibody-antigen binding in the blocked or SWITCH-off state. The team cycled through 22 cycles of staining, imaging, washing and restaining. As Chung explains, the team is working on combining SWITCH and stochastic electrotransport.

"This is an exciting advance as it allows for a systems biology approach to investigating the $3 \mathrm{D}$ relation of numerous targets in a single volume," says Richardson about SWITCH. Imai likes that it helps with labeling because it preserves tissue structure while

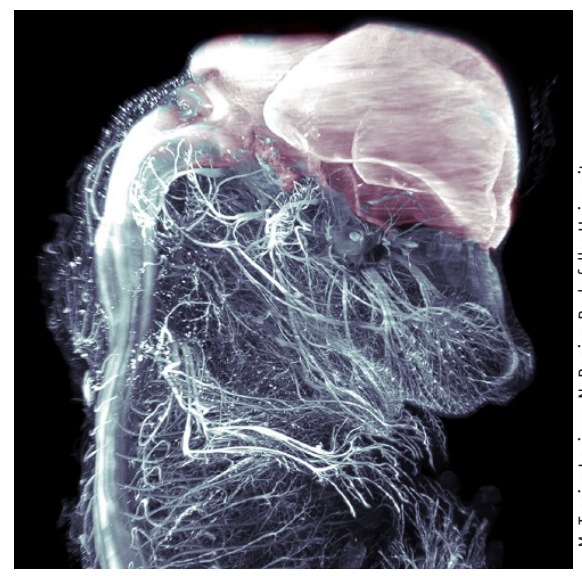

Whole 14-day-old mouse embryo immunostained and cleared with iDISCO to reveal axons. removing lipids. He notes that its use of glutaraldehyde might increase autofluorescence, making it difficult to image thick tissues.

Separately, Richardson sees potential for new infrared (IR) fluorescent probes in cleared tissue even if they are not as bright as certain green and red proteins. With IR dyes, researchers can avoid both Rayleigh scattering, which preferentially scatters blue light, and autofluorescence in the green and red regions of the visible spectrum. "The further you go towards IR, the less scatter there is and the deeper you can image," he says.

Single-molecule mRNA probes are an emerging class of probes to consider. "Wouldn't it be great to expand this from single cells to whole tissues/organs?" asks Richardson. The Deisseroth lab has been working on ways to preserve RNA throughout the CLARITY process, which indicates that such experiments could be attempted in large tissues. Deisseroth says the ongoing work is proceeding well.

\section{Stains and dyes}

Chemical dyes that target specific biomolecules are another potential label type for cleared tissue. Dyes face diffusion issues, too, in cleared tissue, says Imai, but these small molecules will move more quickly than antibodies of typical size.

With the use of dyes in cleared tissue, the question becomes, "Is the target still there?" says Richardson. Lipophilic dyes such as DiI and $\mathrm{DiO}$ will fail if the labeling step is performed after lipid removal. Fixable dye variants perform well, however, if they are crosslinked to protein via aldehydes before lipid solvation and lipid extraction.

The ScaleS team was able to image DiIlabeled neurons in postmortem human brain tissue cleared with ScaleS, says Miyawaki. But the team found that DiI was not compatible with CUBIC, 3DISCO or PACT.

A variety of irreversible ligands could be helpful, says Ellisman, such as bungarotoxinlinked fluorophores. That approach could give researchers a view of all the nicotinic acetylcholine receptors in a cleared sample.

In the lab, Renier says he and his colleagues most commonly use Alexa Fluor dyes conjugated to antibodies, which have delivered the best signal with cleared tissue. Cyanine-based dyes such as $\mathrm{Cy} 3$ also work. "For nuclear stainings, the TO-PRO family of dyes gives the best results so far," he says.

In cleared tissue, Viviana Gradinaru at the California Institute of Technology and her team have labeled opsins with SNAP-tag, 


\section{BOX 2 EXPANSION MICROSCOPY}

Developed in the lab of Edward Boyden at MIT, expansion microscopy, or ExM, expands tissue physically and also renders it transparent ${ }^{9}$. The team infused mouse brain samples with a network of polymers and acrylate that can swell samples 4.5-fold, which delivers a lateral resolution of 60-70 nanometers on an optical microscope. They are exploring greater expansion factorsas high as 15-20-fold-with different chemistries, says Boyden. That would translate to a 20-nanometer lateral resolution.

The team developed a dedicated three-part fluorescent label for ExM. It consists of a chemical fluorophore, a methacryloyl group that can participate in free radical polymerization and thus anchors the tag to the polymer gel, and an oligonucleotide that can hybridize to a sequence that might be attached to an affinity tag or secondary antibody. The label survives the tissue's chemical treatment; it can be targeted to a biomolecule and stays anchored in the polymer network of the expanded sample. They synthesized the label with three different fluorophores to allow multicolor ExM.

Boyden and his team hope to apply many probes in succession to serially read out information, he says. ExM surrounds anchored biomolecules with a homogeneous aqueous environment that is potentially amenable to serial multiplexed tag exchanges. For example, their experiments involve anchoring native RNA- and antibody-targeted DNA barcodes, then doing rapid serial in situ hybridization using probes against these nucleic acid sequences.

In addition to using antibodies as probes for proteins, the team is exploring tags with which to probe lipids and other biomolecules. It might even be possible to perform in situ RNA sequencing.

Tessier-Lavigne thinks that ExM will facilitate imaging of dense labels, such as synaptic markers or markers of dense nerve fibers. But the technique, he says, might be best used in thin preparations. It addresses ways to improve resolution as opposed to clearing to image deeply into samples. Ellisman looks forward to exploring ExM but notes, "It remains to be determined to my satisfaction whether or not ExM expands uniformly." He hopes researchers will now begin to compare current tissue-clearing methods and ExM, perhaps by starting with known molecular targets and known distances and studying what occurs on a molecular level in ExM. Some structures might not expand easily. For example, he says, a synapse does not pull apart well; the pre- and postsynaptic membranes are held together by a kind of "molecular Velcro."

Labeling and imaging the entire mouse brain at the molecular scale will be especially challenging with this technique; it is roughly equivalent to enlarging the mouse brain to the size of the continent of North America, says Ellisman. "That's the tyranny of scale," he says. Dodt is concerned that samples become more gel-like as they expand, which can lead to unsharp images. The Boyden team is working on ways to stiffen samples. Dodt sees ExM as an attractive idea that is set to mature and that advances light microscopy by engineering the sample instead of the microscopes. a polypeptide tag that has been engineered to covalently bind to small fluorescent dyes. The SNAP-tag's multicolor fluorescent probes are good for use in cleared tissue, she says, because the probes can move quickly through cleared tissue. As Richardson explains, SNAP and CLIP tags and similar technologies could be "a solid alternative to immunostaining for quenched fluorescent proteins in solvent-cleared tissue."

SNAP-tags are promising, agrees Kai Johnsson at École Polytechnique Fédérale de Lausanne, adding that mice can be made to express SNAP-tagged proteins. He also believes his near-IR silicon-rhodamine probes (SiRs) are well suited for cleared tissue given that they are fluorogenic, have high permeability and fluoresce only with bright far-red wavelengths of light when bound to a target. Beyond SiRs, he generally sees much potential to stain cleared tissue with small molecule-based probes.

It is advantageous that SNAP-tags are resistant to the harsh preparation techniques used in tissue clearing, says Paul Heppenstall, a researcher at European Molecular Biology Laboratory in Monterotondo, Italy. The probes are small and show good penetration. He and his team have tested SNAP-tags and a SiR on mouse skin tissue and spinal cord samples cleared with a number of techniques-BABB, DBE, methyl salicylate, Scale and SeeDB-and found the staining intensity to be essentially unaltered even with the harshest reagents. In doing this work, he was pleasantly surprised to find that the SNAP protein also works after fixation.

With dyes, labs will want to avoid blue and near-UV wavelengths, says Richardson. Although small-molecule stains such as DAPI and Hoechst may seem like good choices, given that some remaining light scatter is wavelength dependent, these dyes do not perform well in cleared tissue, he says. Substitution with a far-red nuclear dye such as the carbocyanine dye TO-PRO or the farred DNA stain DRAQ5 is preferred, says Richardson.

\section{Consider the optics}

Even when tissue is not pristinely cleared, labs can image half a millimeter or a millimeter deep, says Scott Olenych, who manages product marketing for light-sheet microscopy at Zeiss. At greater depths, imaging can become hazy. Not only can clearing and fixation affect fluorescence, but insufficiently cleared components in a sample can cause a refractive index mismatch, which can affect both brightness and resolution. "We see the frustration on people's faces when they come to us," he says of researchers who bring samples to the Zeiss lab that are "clear-ish." He and his team help troubleshoot, and in some cases they might re-create experiments with a given lab's sample, reagents and immunolabels.

In both cleared and uncleared tissue, light-sheet microscopes with side illumination sometimes cast a shadow called a stripe artifact, says Olenych. The shadow's dimensions depend on factors such tissue density. The stripe can be avoided by altering the angle of the light sheet in a fast up-and-down motion. Such a pivot scanner is built into Zeiss's light-sheet microscopes. Home-built systems, however, might not readily resolve stripe artifacts, he says. Pivot scanners call for a second galvanometer, more optics, mirrors and control software, making them challenging to build.

Adaptive optics techniques, which helped remedy the Hubble Space Telescope's blurred vision, can sharpen images by analyzing and correcting for inhomogeneous light scatter and aberration. These techniques are not yet built into commercial microscopes, says Olenych, but he notes 
that some researchers are developing adaptive optics systems for microscopy.

Brendan Brinkman, who is in the product-planning department at Olympus, also sees labs exploring varied labeling techniques with cleared samples. Small chemical dyes are one emerging option that might prove fruitful, he says. Their successful application will depend on the dyes' relative refractive and absorptive properties, as well as on the ability to use them for specific labeling. An additional issue he sees is that when labels are too dense, it can become difficult to image at depth. Brinkman says that the Chung lab's immunolabel-dispersion approach seems promising because it can address the challenges of uneven staining and label penetration in thick, intact tissues.

Dodt and his team have begun building a prototype of a microscope to optimize imaging of cleared, labeled samples. He previously developed a light-sheet microscope that is commercialized by LaVision BioTec. The new microscope generates a thinner light sheet, which, he believes, will help scientists more readily localize labels in their samples. And they might also combine it with expansion microscopy (Box 2). His concept involves applying the principle behind stimulated emission depletion microscopy to light-sheet microscopy. "We would like to make a de-exciting light sheet above and below the exciting light sheet," he says. In this instrument, the sheet of light is around 50 nanometers, which is thinner than a traditional one.

This light sheet keeps in the dark all parts of the sample above and below the focal plane and creates data sets from the $x, y$ and $z$ dimensions that are then computationally assembled. In larger mouse brain samples imaged in this way, one

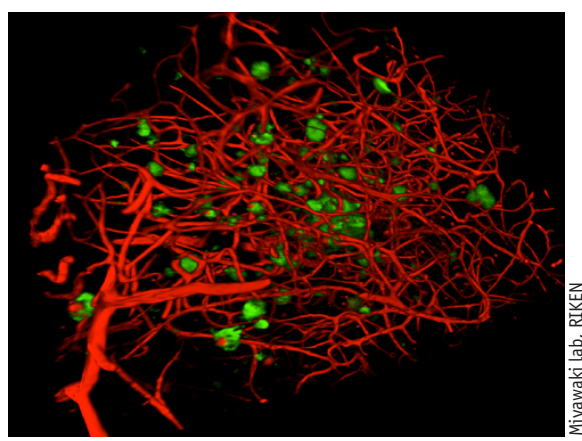

Brain hemispheres of older mice cleared with ScaleS and labeled with Alexa Fluor 488-6E10 and Texas Red-lectin are used to help image amyloid- $\beta$ plaques.
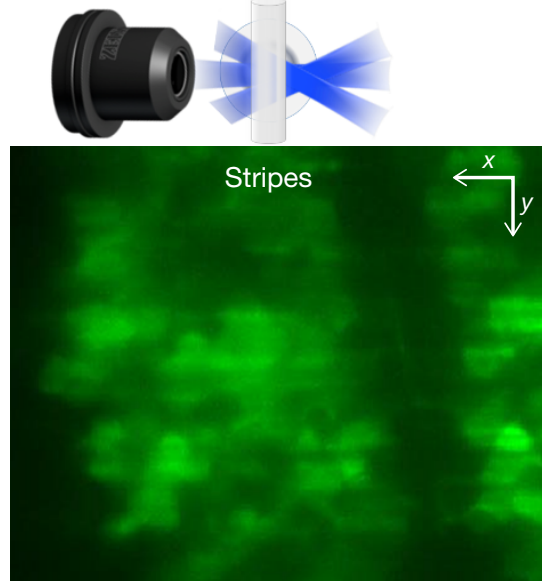
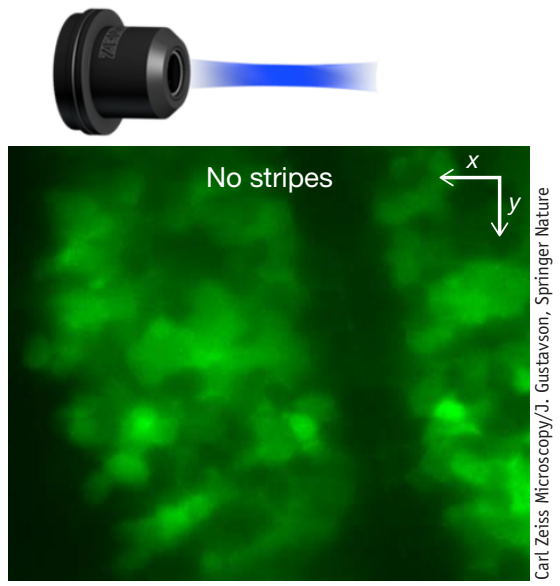

A probe can be obscured by shadows across the cleared tissue in light-sheet microscopy. This stripe artifact can be avoided by pivoting the light sheet up and down quickly.

might be able to capture neuronal populations that are involved in development, learning or memory. For now, says Dodt, one can work with either endogenous fluorescence or externally added labels, but not both.

\section{Path forward}

Broad opportunity beyond neuroscience awaits for labeled, cleared tissues, says Dodt. The next wave, he says, will be biomedicine, probably starting with cancer. He and colleagues are working with cleared breast cancer samples and whole, cleared mice. One day, he says, pathology labs might routinely use tissue clearing and labeling on samples from model organisms and patients.

Clearing and better tissue labeling methods are undoubtedly useful, says Ellisman, but these considerable advances cannot fulfill big dreams just yet. One such dream is imaging all of the proteins in a mouse brain or even a human brain. Immunofluorescence has progressed far from its 1970s beginnings, but fluorescence still cannot be used to gauge the number of proteins expressed at a given brain location, he says. And, of course, other puzzles in neuroscience are harder still, such as understanding how the brain of a person who had Alzheimer's disease reached its plaque-ridden state.

What would help researchers tackle big challenges in neuroscience, says Ellisman, is a high-throughput large-data project to map how many of which proteins are where in the brain, similar to the Allen Institute's gene expression atlases. In cleared tissue, researchers could see, for example, which proteins are dendritic or axonal and more. "We need the proteins because the messenger RNA is not the actor," he says. "The proteins do the work."

Clearing methods can help make this kind of project feasible, says Ellisman, along with a mechanized approach to stain, align and archive the data, perhaps starting with a small volume of tissue. This resource could be based on gene-edited mice in which each gene has been replaced with one bearing a marker so scientists can image the protein that the mRNA encodes. “Then you'll have a normatively expressed marker protein," he says. A key part of this dream project is having on hand the probe technology that works for the diversity of analytical methods.

Clearing techniques and the probes that can be used with them are steadily improving, which means researchers stand to gain much from their ever deeper view into tissues. This gaze leads closer toward the enigmatic goal of characterizing tissue, whole organs and even entire bodies, inside and out.

1. Hama, H. et al. Nat. Neurosci. 14, 1481-1488 (2011).

2. Ertürk, A. et al. Nat. Protoc. 7, 1983-1995 (2012).

3. Chung, K. et al. Nature 497, 332-337 (2013).

4. Ke, M.T., Fujimoto, S. \& Imai, T. Nat. Neurosci. 16, 1154-1161 (2013).

5. Kuwajima, T. et al. Development 140, 13641368 (2013).

6. Renier, N. et al. Cell 159, 896-910 (2014)

7. Richardson, D. \& Lichtman, J. Cell 162, 246257 (2015).

8. Kim, S.Y. et al. Proc. Natl. Acad. Sci. USA 112, E6274-E6283 (2015).

9. Chen, F., Tillberg, P.W. \& Boyden, E.S. Science 347, 543-548 (2015).

Vivien Marx is technology editor for

Nature Methods (v.marx@us.nature.com). 